\title{
An Example of Absence of Turbulence for Any Reynolds Number: II*
}

\author{
C. Marchioro
}

Dipartimento di Matematica, Universitá di Roma I, "La Sapienza", Piazzale A. Moro 5, I-00185 Roma, Italy

\begin{abstract}
We study a viscous incompressible fluid moving in a two dimensional flat torus $[0, L] \times[0,2 \pi], L<2 \pi$. We show a set of external forces for which the stationary state is attractive for any Reynolds number $R$. Moreover, the size of this set and the basin of attraction are independent of $R$.
\end{abstract}

In a previous paper [1] we have considered a viscous incompressible fluid moving in a two dimensional flat torus $[0, L] \times[0,2 \pi], L \leqq 2 \pi$. We have shown an external force $\mathbf{f}_{0}$ for which there is a globally attractive stationary state for any Reynolds number $R$. Moreover, we proved that this stability property holds also for a neighbourhood of $\mathbf{f}_{0}$ of size depending on $R$ (and vanishing for $R \rightarrow \infty$ ). In the present paper we demonstrate that actually for $L<2 \pi$ the size of this neighbourhood is independent of $R$.

The Navier-Stokes equations governing the motion are

$$
\begin{gathered}
\partial_{t} \mathbf{u}+(\mathbf{u} \cdot \nabla) \mathbf{u}=-\nabla p+\mathbf{f}+v \Delta \mathbf{u}, \quad \mathbf{u}(0)=0, \\
\partial_{x} u_{x}+\partial_{y} u_{y}=0, \\
\int_{D} \mathbf{u} d \mathbf{x}=0 ; \quad \int_{D} \mathbf{f} d x=0, \\
D=[0, L] \times[0,2 \pi] ; \quad \mathbf{x}=(x, y)=x \mathbf{c}_{1}+y \mathbf{c}_{2} \in D,
\end{gathered}
$$

where $\mathbf{u}(x, t)$ is the velocity, $p(x, t) \in R^{+}$the pressure, $v>0$ the viscosity, $\mathbf{f}(x)$ the external force. All functions involved are periodic of period $L$ in $x$ and $2 \pi$ in $y$.

We introduce the vorticity $\omega=\partial_{x} u_{y}-\partial_{y} u_{x}$.

Equation (1) becomes

$$
\partial_{t} \omega+(\mathbf{u} \cdot \nabla) \omega=F+v \Delta \omega
$$

where

$$
F=\partial_{x} f_{y}-\partial_{y} f_{x} .
$$

\footnotetext{
* Research partially supported by Italian CNR and Ministero della Pubblica Istruzione
} 
In our problem we want to introduce a reasonable Reynolds number. In [1] we fixed a time scaling and we considered the ratio of the external force and the viscosity. It was enough to prove the existence of a set of stationary states attractive for any Reynolds number. Here we want to prove the independence of the size of this set and so we need more care in defining the Reynolds number (that is the explicit time scale). In general the Reynolds number $R$ is defined as

$$
R=L U / v
$$

where $L$ and $U$ are length and velocity characteristic of the system. We can assume $L$ as the length of the short edge of the torus and $U$ as the supremum of the velocity of the stationary state $\overline{\mathbf{u}}$

$$
R=\sup _{\mathbf{x} \in D} L|\overline{\mathbf{u}}(\mathbf{x})| v .
$$

The link between the stationary state and the external force will be discussed at the end of the paper.

It is well known that in general the behavior of the solutions depends on $R$. When $R$ is small there exists an attractive stationary state; when $R$ increases this state loses its stability and for large $R$ the motion becomes chaotic. This behavior is related with turbulence (see for instance [2]).

In this paper we want to show an open set of stationary states which are attractive for any $R$ with a basin of attraction independent of $R$.

We write the stationary state $\overline{\mathbf{u}}(x, y)$ as

$$
\mathbf{u}(x, y)=\overline{\mathbf{u}}_{1}(y)+\overline{\mathbf{u}}_{2}(x, y),
$$

where

$$
\begin{gathered}
\overline{\mathbf{u}}_{1}(y)=\mathbf{c}_{1}\left(A_{1} \cos y+A_{2} \sin y\right), \\
A=\max \left(\left|A_{1}\right|,\left|A_{2}\right|\right),
\end{gathered}
$$

$\mathbf{u}_{2}(x, y)=$ all other terms in the Fourier development.

Hence $\bar{\omega}=\omega_{1}+\omega_{2}$, where

$$
\omega_{1}=A_{1} \sin y-A_{2} \cos y, \quad \omega_{2}=\partial_{x} \bar{u}_{2 y}-\partial_{y} \bar{u}_{2 x} .
$$

We define

and

$$
\begin{gathered}
\mathbf{v}=\mathbf{u}-\overline{\mathbf{u}} ; \quad E=1 / 2 \int_{D} \mathbf{v}^{2} d \mathbf{x}, \\
\delta=\omega-\bar{\omega} ; \quad N=1 / 2 \int_{D} \delta^{2} d \mathbf{x},
\end{gathered}
$$

$$
\int\left(\nabla \bar{\omega}_{2}\right)^{2} d \mathbf{x}=W^{2} v^{2}
$$

We discuss the relation between $R, v$, and $A$. Of course, $v$ is a scaling parameter on the velocity field. We can fix $v=1$ and use sup $|\overline{\mathbf{u}}|$ as the quantity proportional to the Reynolds number. Moreover, $R>L A$, so that a property valid for any $A$ holds for $R$ as large as we want. From here on $c_{i}$ means constant independent of $A$. 
The result of this paper is stated in the following theorem:

Theorem. For every $A$ and $W<c_{1}$ the stationary state attracts exponentially each solution such that $(N(0)-E(0))<c_{2}$. More precisely

$$
\begin{aligned}
& E(t)=1 / 2 \int_{D} \mathbf{v}^{2} d \mathbf{x} \rightarrow 0 \quad \text { exponentially, } \\
& N(t)=1 / 2 \int_{D} \delta^{2} d \mathbf{x} \rightarrow 0 \quad \text { exponentially. }
\end{aligned}
$$

Proof. We introduce the stream function $\psi=\bar{\psi}+\varphi$,

$$
\nabla^{\perp} \psi=\mathbf{u} ; \quad \nabla^{\perp}=\left(\partial_{y},-\partial_{x}\right) ; \quad \nabla^{\perp} \varphi=\mathbf{v},
$$

and we develop it in Fourier series:

We define

$$
\begin{aligned}
\varphi(x, y)= & \sum_{m=0}^{\infty} \sum_{n=0}^{\infty}\left\{a_{m n} \cos (m h x) \cos (n y)+b_{m n} \cos (m h x) \sin (n y)\right. \\
& \left.+c_{m n} \sin (m h x) \cos (n y)+d_{m n} \sin (m h x) \sin (n y)\right\} .
\end{aligned}
$$

$$
\begin{aligned}
E_{2}= & 1 / 2 \int\left(\mathbf{v}_{2}\right)^{2} d \mathbf{x}=[(\pi L) / 2] h^{2}\left[\left(a_{10}\right)^{2}+\left(c_{10}\right)^{2}\right] \\
& +[(\pi L) / 4] \sum_{m=1}^{\infty} \sum_{n=1}^{\infty}\left(n^{2}+h^{2} m^{2}\right)\left[\left(a_{m n}\right)^{2}+\left(b_{m n}\right)^{2}+\left(c_{m n}\right)^{2}+\left(d_{m n}\right)^{2}\right], \\
& N_{1}=1 / 2 \int\left(\delta_{1}\right)^{2} d \mathbf{x}=[(\pi L) / 2]\left[\left(a_{01}\right)^{2}+\left(b_{01}\right)^{2}\right], \\
N_{2}= & 1 / 2 \int\left(\delta_{2}\right)^{2} d \mathbf{x}=[(\pi L) / 2] h^{4}\left[\left(a_{10}\right)^{2}+\left(c_{10}\right)^{2}\right] \\
& +[(\pi L) / 4] \sum_{m=1}^{\infty} \sum_{n=1}^{\infty}\left[\left(a_{m n}\right)^{2}+\left(b_{m n}\right)^{2}+\left(c_{m n}\right)^{2}+\left(d_{m n}\right)^{2}\right]\left(h^{2} m^{2}+n^{2}\right)^{2},
\end{aligned}
$$

and so

$$
E=E_{1}+E_{2} ; \quad N=N_{1}+N_{2} .
$$

By a direct calculation we have

and hence

$$
\begin{aligned}
& \frac{d N}{d t}=-\int \delta(\mathbf{v} \cdot \nabla) \bar{\omega} d \mathbf{x}-\int(\nabla \delta)^{2} d \mathbf{x}, \\
& \frac{d E}{d t}=-\int \mathbf{v} \cdot(\mathbf{v} \cdot \nabla) \overline{\mathbf{u}} d \mathbf{x}-\int(\nabla \mathbf{v})^{2} d \mathbf{x}
\end{aligned}
$$

$$
\begin{aligned}
\frac{d(N-E)}{d t}= & -\int\left[\delta(\mathbf{v} \cdot \nabla) \bar{\omega}_{2}-\mathbf{v} \cdot(\mathbf{v} \cdot \nabla) \bar{u}_{2}\right] d \mathbf{x}-\int\left[(\nabla \delta)^{2}-(\nabla \mathbf{v})^{2}\right] d \mathbf{x} \\
= & -\int\left[\delta_{1}\left(\mathbf{v}_{2} \cdot \nabla\right) \bar{\omega}_{2}+\delta_{2}\left(\mathbf{v}_{1} \cdot \nabla\right) \bar{\omega}_{2}+\delta_{2}\left(\mathbf{v}_{2} \cdot \nabla\right) \bar{\omega}_{2}-\mathbf{v}_{1} \cdot\left(\mathbf{v}_{2} \cdot \nabla\right) \overline{\mathbf{u}}_{2}\right. \\
& \left.-\mathbf{v}_{2} \cdot\left(\mathbf{v}_{1} \cdot \nabla\right) \overline{\mathbf{u}}_{2}-\mathbf{v}_{2} \cdot\left(\mathbf{v}_{2} \cdot \nabla\right) \overline{\mathbf{u}}_{2}\right] d \mathbf{x}-\int\left[(\nabla \delta)^{2}-(\nabla \mathbf{v})^{2}\right] d \mathbf{x} .
\end{aligned}
$$


We note that

$$
\begin{aligned}
N-E= & N_{2}-E_{2}=[(\pi L) / 2] h^{2}\left(h^{2}-1\right)\left[\left(a_{10}\right)^{2}+\left(c_{10}\right)^{2}\right] \\
& +[(\pi L) / 4] \sum_{m=1}^{\infty} \sum_{n=1}^{\infty}\left[\left(a_{m n}\right)^{2}+\left(b_{m n}\right)^{2}+\left(c_{m n}\right)^{2}+\left(d_{m n}\right)^{2}\right] \\
& \times\left(h^{2} m^{2}+n^{2}\right)\left(h^{2} m^{2}+n^{2}-1\right) .
\end{aligned}
$$

All terms in the sum are positive and hence $(N-E)$ small implies that all coefficients in the Fourier development different from $a_{01}$ and $b_{01}$ must be small.

We study now the evolution in time of $E_{1}$ :

$$
\frac{d E_{1}}{d t}=-\int \mathbf{v}_{1} \cdot\left[\left(\mathbf{v}_{2} \cdot \nabla\right) \overline{\mathbf{u}}_{2}+\left(\mathbf{v}_{2} \cdot \nabla\right) \mathbf{v}_{2}+\left(\overline{\mathbf{u}}_{2} \cdot \nabla\right) \mathbf{v}_{1}\right] d \mathbf{x}-2 E_{1} .
$$

We observe that

$$
\begin{aligned}
& \int\left[(\nabla \delta)^{2}-(\nabla \mathbf{v})^{2}\right] d \mathbf{x}=\pi L h^{4}\left(h^{2}-1\right)\left[\left(a_{10}\right)^{2}+\left(c_{10}\right)^{2}\right] \\
& \quad+\sum_{m=1}^{\infty} \sum_{n=1}^{\infty}(\pi L / 2)\left[\left(a_{m n}\right)^{2}+\left(b_{m n}\right)^{2}+\left(c_{m n}\right)^{2}+\left(d_{m n}\right)^{2}\right]\left[h^{2} m^{2}+n^{2}\right] \\
& \quad \times\left[(m h+n)^{2}-1\right] \geqq 2\left(h^{2}-1\right)(N-E) .
\end{aligned}
$$

Then we estimate the other terms in (10) and (13). By use of the definition of $E_{1}$, the expression (11), integration by parts, we obtain

$$
\begin{gathered}
\frac{d(N-E)}{d t} \leqq c_{3} W\left(E_{1}\right)^{1 / 2}(N-E)^{1 / 2}+c_{4} W(N-E)-c_{5}(N-E) \\
\frac{d E_{1}}{d t} \leqq c_{6} W\left(E_{1}\right)^{1 / 2}(N-E)^{1 / 2}+c_{7}\left(E_{1}\right)^{1 / 2}(N-E)-2 E_{1}
\end{gathered}
$$

We study these inequalities. We suppose initially

$$
c_{8}(N-E)^{1 / 2}<W c_{6} .
$$

We prove that this property holds for every time if $W$ is small enough. In fact, we sum (14) and (15), and we have

$$
\frac{d\left[(N-E)^{1 / 2}+\left(E_{1}\right)^{1 / 2}\right]}{d t} \leqq c_{9} W\left[(N-E)^{1 / 2}+\left(E_{1}\right)^{1 / 2}\right]-c_{10} W\left[(N-E)^{1 / 2}+\left(E_{1}\right)^{1 / 2}\right]
$$

For $W$ small enough $(N-E)^{1 / 2}+\left(E_{1}\right)^{1 / 2}$ is bounded by a function which vanishes exponentially. So $E_{1}$ and $(N-E)$ separately vanish exponentially.

Finally, we want to relate the stationary state with the external force $\mathbf{f}$

where

$$
\mathbf{f}(\mathbf{x}, y)=\mathbf{f}_{1}(y)+\mathbf{f}_{2}(x, y),
$$

$$
\mathbf{f}_{1}(x)=\mathbf{c}_{1}\left(g_{1} \cos y+g_{2} \sin y\right),
$$

$\mathbf{f}_{2}(x, y)=$ other terms in the Fourier development

$$
F=F_{1}+F_{2},
$$


where

$$
\begin{aligned}
& F_{1}=\partial_{x} f_{1 y}-\partial_{y} f_{1 x}, \\
& F_{2}=\partial_{x} f_{2 y}-\partial_{y} f_{2 x} .
\end{aligned}
$$

Proceeding as in [1] we have

$$
\int\left[\bar{\omega}_{2} F_{2}-\overline{\mathbf{u}}_{2} \cdot \mathbf{f}_{2}\right] d \mathbf{x}=\int\left[\left(\nabla \bar{\omega}_{2}\right)^{2}-\left(\nabla \overline{\mathbf{u}}_{2}\right)^{2}\right] d \mathbf{x} .
$$

Hence

$$
\begin{aligned}
c_{11} \int\left(\nabla \bar{\omega}_{2}\right)^{2} d \mathbf{x} & \leqq \int\left[\left(\nabla \bar{\omega}_{2}\right)^{2}-\left(\nabla \overline{\mathbf{u}}_{2}\right)^{2}\right] d \mathbf{x} \leqq\left[\int\left(\bar{\omega}_{2}\right)^{2} d \mathbf{x}\right]^{1 / 2}+\left[\int\left(\overline{\mathbf{u}}_{2}\right)^{2} d \mathbf{x}\right]^{1 / 2} \\
& \leqq\left[\int\left(\nabla \bar{\omega}_{2}\right)^{2} d \mathbf{x}\right]^{1 / 2}\left\{\left[\int\left(F_{2}\right)^{2} d \mathbf{x}\right]^{1 / 2}+\left[\int\left(\mathbf{f}_{2}\right)^{2} d \mathbf{x}\right]^{1 / 2}\right\},
\end{aligned}
$$

and so

$$
c_{11}\left[\int\left(\nabla \bar{\omega}_{2}\right)^{2} d \mathbf{x}\right]^{1 / 2}<\left[\int\left(F_{2}\right)^{2} d \mathbf{x}\right]^{1 / 2}+\left[\int\left(\mathbf{f}_{2}\right)^{2} d \mathbf{x}\right]^{1 / 2} .
$$

Then we see that small forces $\mathbf{f}_{2}$ produce small stationary states, $\mathbf{u}_{2}$ for any $\mathbf{f}_{1}$.

\section{References}

1. Marchioro, C.: An example of absence of turbulence for any Reynolds number. Commun. Math. Phys. 105, 99 (1986)

2. Joseph, D.D.: Stability of fluid motion, Vols. I, II. Berlin, Heidelberg, New York: Springer 1976

Communicated by J. L. Lebowitz

Received May 13, 1986 
